\title{
Wind turbine systems operational state and reliability evaluation: An artificial neural network approach
}

\author{
D. O. Aikhuele ${ }^{\mathrm{a}}$, A. Periola ${ }^{\mathrm{b}}$ and D. E. Ighravwe ${ }^{\mathrm{a}^{*}}$
}

${ }^{a}$ Department of Mechanical and Biomedical Engineering, Bells University of Technology, Ota, Nigeria ${ }^{b}$ Department of Electrical and Computer Engineering, Bells University of Technology, Ota, Nigeria

\section{H R O N I C L E}

\section{Article history:}

Received: December 28, 2018

Received in revised format: May

4, 2019

Accepted: May 4, 2019

Available online: May 4, 2019

Keywords:

Wind turbine systems

Artificial neural network

Downtime

System reliability

\section{A B S T R A C T}

\begin{abstract}
The increased role of wind turbine systems makes it important for its operational states to be continuously monitored and optimized. This goal can be achieved using existing methods, which relies on closed-form expressions. The use of these methods, however, becomes challenging when interacting parameters cannot be fully presented with closed form expressions. In this paper, an artificial neural network (ANN) based algorithm is proposed as a solution to this problem. This algorithm is used to estimate wind turbine systems operational state and reliability. The proposed method is able to provide a more holistic approach to manage a wind turbine system with respect to the problem mentioned above. Simulation results show that the developed ANN can predict the average number of failures per year, distribution of failure and average downtime per failure with good accuracy. This was achieved using an ANN model with 5-15-3 architecture. The model generates mean square errors of $4.6 \times 10^{-3}, 4.2 \times 10^{-3}$, and $4.0 \times 10^{-3}$ at the training, validation, and testing stages, respectively. The study is beneficial to wind turbine practitioners and manufacturers as its findings can provide in-depth understandings of reliability issues of the system.
\end{abstract}

\section{Introduction}

Renewable wind energy is an important alternative power source for homes and industries. It reduces the dependence on traditional power sources that cause pollution and global warming (Hoel \& Kverndokk, 1996). The desire to push for more reduction in the use of traditional energy sources has caused an increased in the use of wind power technologies. These technologies have necessitated the design of mechanisms that can enhance its reliability. Reliability, in this case, can be enhanced by the execution of maintenance and repairing scheme. Maintenance and repair include inspection (failure detection), re- 
build, alteration and the supply of spare parts, accessories and the provision of a healthy reliability management scheme for wind turbine systems. A key requirement of reliability management in wind turbines is the task of a prior detection of failures. This is necessary to ensure reliable continuous operation and maintenance of wind turbine machine systems (Kusiak \& Li, 2011; Aikhuele \& Turan, 2016, 2018). A priori failure detection in wind turbine is a prerequisite for ensuring failure prediction maintenance (Nivedh, 2014). Furthermore, the idea of failure diagnosis in wind turbine systems is to detect the location of the exact fault, as well as to determine its extent in the systems (Alwi et al., 2011). Fault detection in wind turbine systems is a challenging task because of the inter-related and interaction of its components during operation (Anderson \& Johnson, 1997; Vogus \& Welbourne, 2003). When this system's faults are properly managed, there will be a high chance of supplying home and industries with steady energy.

Fault estimation and failure detection can be described as a decision-making process in many fields of discipline, this includes engineering (Aikhuele, 2018; $\mathrm{Su} \& \mathrm{Hu}, 2018$ ). Fault and failure detection, therefore, provides advanced information about a system; this process helps in the identification and description of the intensity of a system behavior. Faults in wind turbines can occur in a number of parts and components such as their sensors or actuators parts. The proactive detection of faults in this kind of system will save it from unforeseen hazards, and it will improve its performance and reliability management (Gao et al., 2015; Aikhuele et al., 2017). For instance, Tabatabaeipour et al. (2014) used statistical modelling and distribution techniques to model the performance of wind turbine systems. This approach is novel, but it is unsuitable when information about operational context is required. Other approaches which are useful for this system's fault detection are optimization and fuzzy logic models. These models assumed that the nonlinear relationship exists among fault detection variables.

Witczak and Rotondo (2015) used a Takagi-Sugeno to model a wide class of nonlinear wind turbine systems, the model was used also for designing a robust unknown input observer (UIO) and for fault estimation. Witczak et al. (2017) used a combination of adaptive and parameter estimation schemes that assumes a set of possible faults affecting the dynamics of the wind turbine. Awedni and Krichen (2016) proposed an adaptive fault diagnosis observer method for enhancing the accuracy of fault estimation. Simani and Farsoni (2018) reported a data-driven approach for robust fault diagnosis in wind turbine systems. Their approach was able to estimate turbines fault, even in the presence of noisy measurements. Mensah and Dueñas-Osorio (2012) proposed a closed-form method for reliability and failure consequence evaluation of wind turbine system using a Monte Carlo simulation approach.

Chronological multiple state probability model of tidal power generation system (TPGS) has, also, been used to estimate this system's fault and failure detection (Mingjun et al., 2016). It is a method that considers both the forced outage rate of the TPGS and the random nature of tidal current speed for reliability evaluation. Aval and Ahadi (2016) used a fault tree method (FTA) to estimate wind turbines reliability. Kahrobaee and Asgarpoor (2009) presented a Markov analytic method for time-based reliability assessment of wind turbine system; the work considered wind speed, failure, and repair rates as system's parameters.

Most of the cited works above-considered wind turbines reliability at a single level, to provide an increased power output, this system reliability should be considered at two or multiple levels; this is still a challenge for existing mechanisms such as ant colony algorithm and genetic algorithm (Wang et al., 2004; Bianchi \& Dorigo., 2006; Blinco et al., 2014). Some of the attempts that have been reported when these algorithms were used to solve this problem were computationally expensive, especially when different combinations of multiple parameters were considered. Also, these methods, require the interacting variables used in the fault estimation to be related by a closed form expression, which is practical, not feasible for all operating parameters. To this end, it is important to provide a suitable alternative method that addresses these challenges. In this study, a two-stage process for fault detection and failure estimation in wind turbine systems is proposed. The two-stage process which includes fault prediction and 
failure estimation was conducted within the context of a wind turbine project in Nigeria. The study contributes to the body of knowledge by providing an alternative method to detect faults and estimate failure in wind turbine system using artificial neural network (ANN) model. This model is considered suitable because it encodes available operational data into matrices provided by its layers as specified in its architecture. Hence, it can be used to realize the best system configuration in cases where the interacting variables are not related by a closed-form expression.

The rest of the paper is organized as follows; in section 2, the concept of ANN is introduced. The structural analysis and the typical faults of wind turbine system are discussed in Section 3. In section 4, a numerical case study of the wind turbine is presented. The concluding remarks are given in section 5.

\section{Artificial Neural Networks}

ANN models have found application in several areas in wind turbine modelling. And their performance has been used for fault identification and estimation, prediction and optimization of the system architecture, classification, forecasting and evaluation, as well as in systems control systems (Ata, 2015). These models are computational models motivated by the human brain. They accept known input variables and uses them to determine or predict expected output variables. The variables describe the operational parameters of the system being modelled. They can be designed using different architectures such as selforganizing map, echo state networks, liquid state machines and feedforward neural networks (Bai et al., 2018). Recently, the deep learning ANN models have received significant attention in the research community due to their successes in pattern and image recognition.

They are suitable for real-world problems where the development of a mathematical model relating inputs and outputs proof cumbersome and challenging. These models comprise three layers - input, hidden and output layers- a sample of these models is shown in Figure 1. The hidden layer encodes the unknown mathematical expression that links the input and output parameters in a problem under consideration. The unknown mathematical expression is derived using a training procedure (Periola and Falowo, 2017). During training, the input layer is assigned an input layer activation function, and it accepts pre-processed input parameters. The hidden layer and output layer also have their own activation functions. The training of these models is completed when their error functions, which could be their mean square errors (MSE), reaches the target value (Zheng \& Ming, 2017).

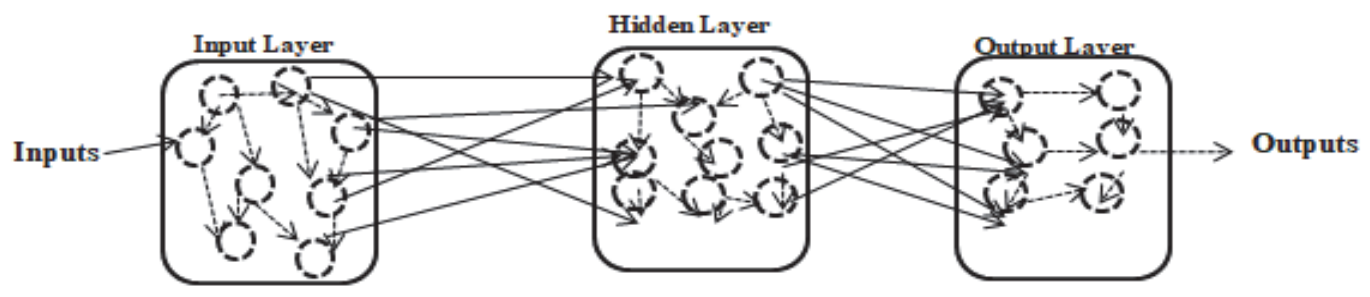

Fig. 1. Illustration of an Artificial Neural Network

\section{The structural analysis, and the typical faults in wind turbine system}

Wind turbine technology has continued to improve from its rise to the present. This technology has continuously been updated as well as the varieties of the wind units. Although the components in this system made by manufacturers appear to be different in their shape and size, their structures are always similar (Chengbing \& Xinxin, 2012). A typical wind turbine system is shown in Fig. 2. These systems can be categorized as fixed pitch stall-type, pitch stall-type, variable speed and the constant frequency type. The wind units are manufactured depending on the choice or the design of a manufacturer. These systems are 
composed of the following main components wind rotor, gearbox, pitch system and among others. These systems common faults are within the following components:

i. Wind rotor: The wind rotor which is made either from wooden materials, fiberglass or metals, collects energy from the wind for the system, they are mainly affected by long-term use and climatic condition.

ii. Gearbox: The main function of the gearbox is to increase the speed of the low-speed shaft of the rotor. This is necessary when the rotor shaft's speed falls short of the requirements of wind turbine generators. Most of the faults in the gearbox are caused by poor lubrication, causing friction and abrasion in the gearbox.

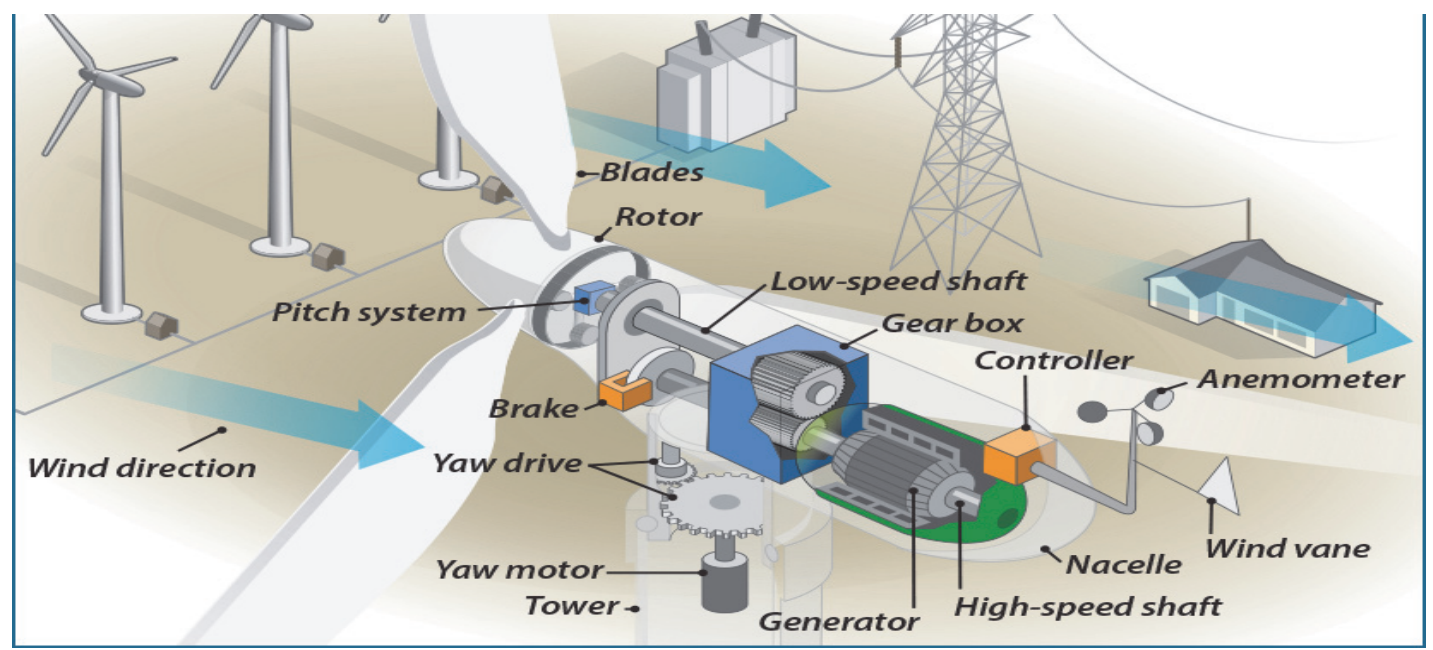

Fig. 2. The structure of a wind turbine

iii. The pitch system: The pitch system regulates and controls the speed of the rotor (power) by changing the angle and direction of the blade, thereby preventing the rotor from turning in winds that are too high or too low to produce electricity for the system. The faults in the Pitch system are classified as 'No pitch fault', potential pitch fault and pitch fault established and.

iv. The wind turbine tower: Wind turbine towers which are made from either tubular steel, steel lattice or concrete are used for supporting the wind turbine structure. The towers are designed mainly to be very tall to allow for the turbines system to capture more energy and to generate more electricity. The towers have a very small probability of failure and are affected mainly by vibration coming from components equipped with sensors and other components affected by weathering condition.

\section{Case Study}

This study considered the use of the wind turbine system for energy generation in Nigeria. For such an application, it is important to quantify the ability of the system to generate energy at its rated capacity. The maximal functioning of the system is dependent on the functional status of its component parts. In achieving this goal, it important to proactively determine the functional status of the wind turbine. This is achieved by predicting the annual average number of failures, failure of distribution, and average downtime per failure. Total downtime per component, average downtime per year, average downtime per year, and a total number of failures per component as inputs parameters were, also, considered. These output and input parameters were selected because they describe the ability of the system with respect to its rated capacity. The ability to proactively estimate the predicted parameters will enable an energy utility company or an energy provider to have a priori knowledge of energy that can be sold to customers. 
A component specific relation between the selected parameters and wind turbine sub-systems is challenging due to the interdependency of the components. This is because the selected parameters have a pervasive effect and affect all parts of an installed wind turbine.

To simulate the required system, neural network architecture in MATLAB was used. An ANN model was is designed to accept the total downtime per component, average downtime per year, average downtime per year per turbine, distribution of downtime and the total number of failed components as inputs. The model's outputs are the average number of failures per year, distribution of failure and average downtime per failure. Other relevant information about the model is presented in Table 1.

Table 1

ANN design and simulation parameters

\begin{tabular}{lc}
\hline Parameter & Value \\
\hline Number of input parameters & 5 \\
Number of output parameters & 3 \\
Number of neurons in the input, hidden and output layer & $5,3,1$ \\
Input, hidden and output layer transfer functions & Tansig, Tansig and Purelin \\
Training algorithm & Adaptive gradient with momentum \\
Target mean square error & 0.0001 \\
\hline
\end{tabular}

The simulation was done using a feed-forward ANN with a single hidden layer. In developing ANNs, the required datasets were divided into three sets: training, validation and testing data sets. The training dataset was used to train the model via the adaptation of the weights between the connections in the input, hidden and output layer neurons. This process was followed by model validation; where the validation data set is combined with some samples of the training datasets and used to test the developed model's accuracy. The testing dataset were used to examine the model's accuracy. The training process is completed when the validation and testing MSE were less than that of the training phase MSE. To achieve this, a model pruning procedure was adopted - gradual reduction of the number of hidden layer neurons

This approach is suitable for a scenario where there is limited availability of computing infrastructure and resources. Hence, the number of neurons in the hidden layer is reduced from 25 to 10 . The training performance is deemed to be well trained because the training MSE is observed to be reduced as the epochs increased, in addition, the validation and testing MSE reduced as the training epochs increased. The results presented in Figure 3 showed that the model convergence when the training MSE was $5.4 \times$ $10^{-2}$. And at this instance, the validation and testing MSE is the same: $4.4 \times 10^{-2}$. The validation and testing procedures demonstrate that the model is well trained. The disparity in the training, validation and testing results is due to the proportion of samples used for training, validation and testing. When the proportion of samples used for validation and testing is 20 and $10 \%$, these results change and they are shown in Figure 4, for this case, the training, validation, and testing MSE are $5.8 \times 10^{-2}, 5.0 \times 10^{-2}$, and $5.6 \times 10^{-2}$, respectively.

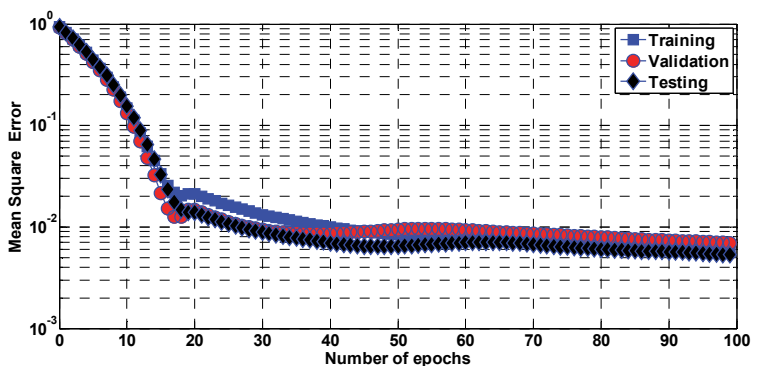

Fig. 3. Performance of the ANN with 25-neurons in the hidden layer for 15 and $10 \%$ validation and testing samples

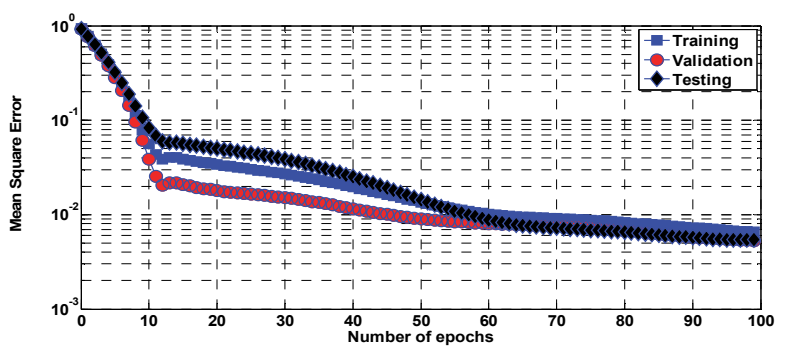

Fig. 4. Performance of the ANN with 25 -neurons in the hidden layer for 20 and $10 \%$ validation and testing samples 
When hidden layer neurons were 20, the training results obtained are shown in Fig. 5, for this case, the proportions of samples used for validation and testing are 15 and 10\% respectively. Training MSE exceeds the validation and testing MSE as shown in Fig. 5. And this signifies that the developed ANN model is able to predict the expected outputs for previously unknown inputs. In this case, the training, validation, and testing MSE are $5.5 \times 10^{-2}, 3.4 \times 10^{-2}$, and $5.7 \times 10^{-2}$, respectively. Also, when the validation and testing sample sizes were changed to 20 and $10 \%$, the MSE for the training and testing MSE reduced to $4.9 \times 10^{-2}$ and $4.3 \times 10^{-2}$, respectively, while the validation MSE increased to $4.1 \times 10^{-2}$, see Fig. 6 for model details.

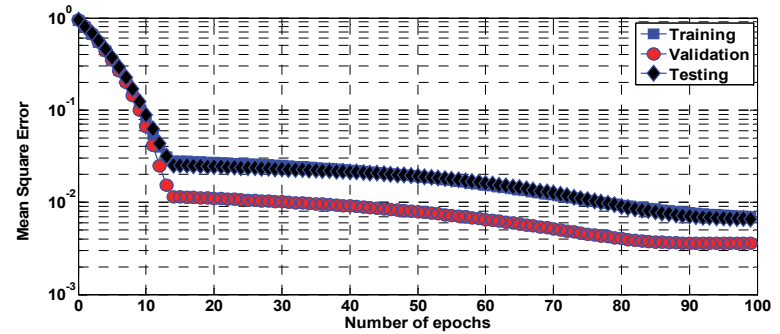

Fig. 5. Performance of the ANN with 20-neurons in the hidden layer for 15 and $10 \%$ validation and testing samples

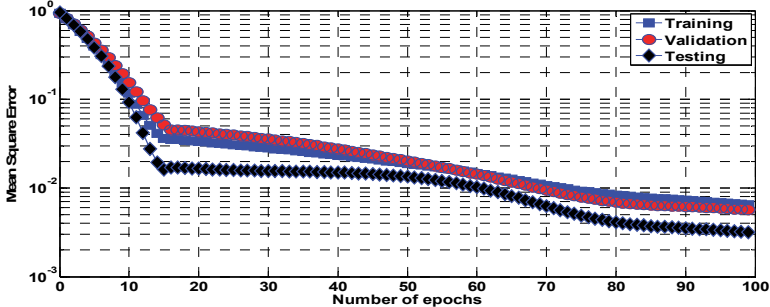

Fig. 6. Performance of the ANN with 20-neurons in the hidden layer for 20 and $10 \%$ validation and testing samples

When a further simulation was carried using a hidden layer with 15 neurons, the proposed model performance improved, the obtained are presented in Fig. 7a and Fig. 7b. The results in Fig. 7a shows the modes' performance for 15 and $10 \%$ validation and testing samples. And the training, validation, and testing MSE are $4.6 \times 10^{-3}, 4.2 \times 10^{-3}$, and $4.0 \times 10^{-3}$, respectively. These attributes of the model generated better results when compared with the same with 20 and $10 \%$ validation and testing samples, respectively. This new model attributes generated training, validation, and testing MSE are $5.3 \times 10^{-3}, 4.3$ $\times 10^{-3}$, and $4.1 \times 10^{-3}$, respectively. Another instance of the model with 10 neurons in the hidden layer was considered. The performance of this model with are shown Fig. 8a for 15 and 10\% validation and testing samples and Fig. 8b for 20 and 10\% validation and testing samples. The results in Fig. 8a generated MSE values of $5.1 \times 10^{-3}, 4.7 \times 10^{-3}$, and $4.2 \times 10^{-3}$ for the training, validation, and testing samples, respectively. The MSE values for the training, validation, and testing samples in Fig. $8 \mathrm{~b}$ are the same as those in Fig. 8a. At this stage, the training of the model was stopped.

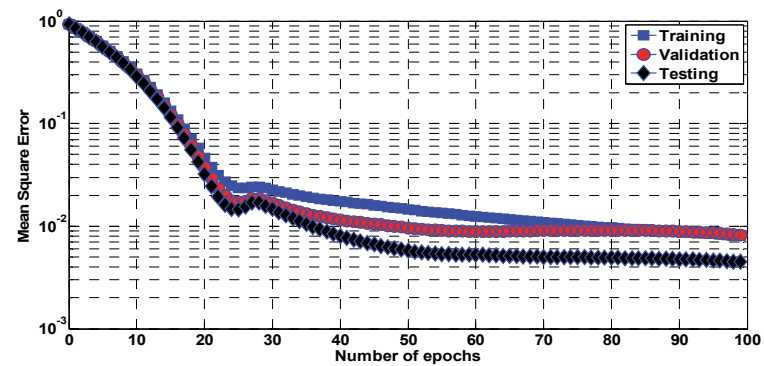

Fig. 7a. Performance of the ANN with 15-neurons in the hidden layer for 15 and $10 \%$ validation and testing samples

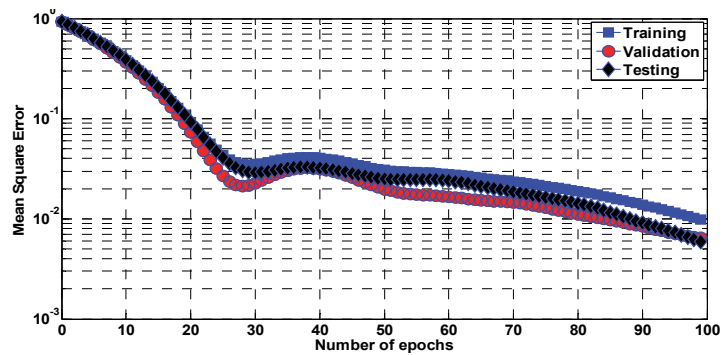

Fig. 8a. Performance of the ANN with 10-neurons in the hidden layer for 15 and $10 \%$ validation and testing samples

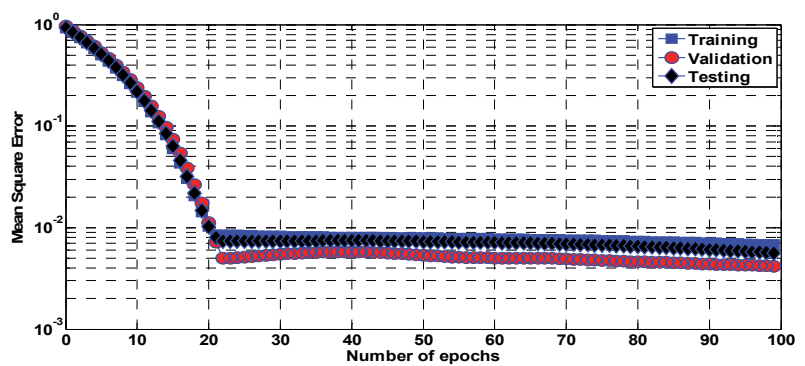

Fig. 7b. Performance of the ANN with 15-neurons in the hidden layer for 20 and $10 \%$ validation and testing samples

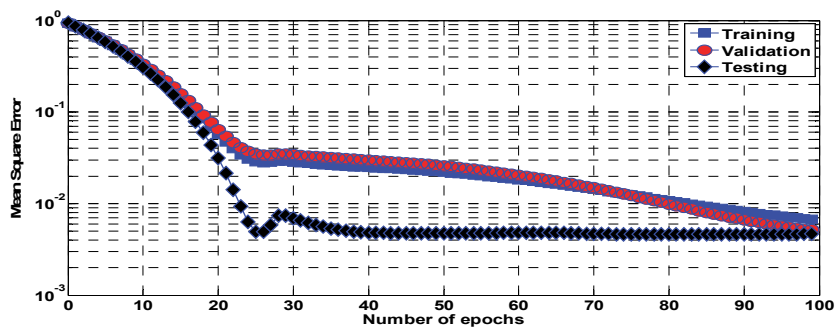

Fig. 8b. Performance of the ANN with 10-neurons in the hidden layer for 20 and $10 \%$ validation and testing samples 
Based on the above results, the most suitable ANN architecture for the case study is the model that generated MSE of $4.6 \times 10^{-3}, 4.2 \times 10^{-3}$, and $4.0 \times 10^{-3}$ at the training, validation, and testing stages, respectively; this is the model with 5-15-3 with a validation and testing samples of 15 and $10 \%$, respectively.

\section{Conclusions}

This paper examines the challenge of estimating the operational state of wind turbine systems. It proposes the use of the ANN in estimating wind turbine system performance. The proposed method differs from existing methods of reliability estimation which are only suited for pre-defined objectives that are not easy to change. In addition, the existing reliability estimation methods require the availability of a closed form expression. The ANN model presented can be used in the absence of a closed form expression. The model is able to realize the best system configuration in cases where the interacting variables are not related by a closed form expression.

The results from the study showed that the developed ANN is able to predict an average number of failures per year, distribution of failure and average downtime per failure with good accuracy for the wind turbine system. Also, it addresses the drawback in the fault estimation methods, where the wind turbine interacting parameters cannot be fully presented or related to closed-form expressions. This study is beneficial to wind turbine manufacturers as its findings can provide design and industrial practitioners with in-depth understanding of reliability issues of this system. And its findings could lead to the introduction of an effective reliability management strategy for wind turbines.

\section{References}

Aikhuele, D. O., \& Turan, F. B. (2016). Intuitionistic fuzzy-based model for failure detection. SpringerPlus, 5(1), 1938.

Aikhuele, D. O., \& Turan, F.M. (2018). A modified exponential score function for troubleshooting an improved locally made Offshore Patrol Boat engine. Journal of Marine Engineering \& Technology, 17(1), 52-58.

Aikhuele, D. O. (2018). Intuitionistic Fuzzy Model for Reliability Management in Wind Turbine System. Applied Computing and Informatics.

Aikhuele, D. O., Sorooshian, S., Ansah, R. H., \& Turan, F. M. (2017). Application of intuitionistic fuzzy TOPSIS model for troubleshooting an offshore patrol boat engine. Polish Maritime Research, 24(2), 68-76.

Alwi, H., Edwards, C., \& Tan, C. P. (2011). Fault tolerant control and fault detection and isolation. In Fault Detection and Fault-Tolerant Control Using Sliding Modes (pp. 7-27). Springer, London.

Anderson, V., \& Johnson, L. (1997). Systems Thinking Basics. From Concepts to Causal Loops. Waltham: Mass Pegasus Comm., Inc.

Ata, R. (2015). Artificial neural networks applications in wind energy systems: a review. Renewable and Sustainable Energy Reviews, 49, 534-562.

Aval, S. M. M., \& Ahadi, A. (2016). Reliability evaluation of wind turbine systems' components. Bulletin of Electrical Engineering and Informatics, 5(2), 160-168.

Awedni, O., \& Krichen, L. (2016, April). Adaptive observer-based fault estimation for a DFIG based wind turbine system. In Ecological Vehicles and Renewable Energies (EVER). 2016 Eleventh International Conference on (pp. 1-7). IEEE.

Bai, Y., Sun, Z., Deng, J., Li, L., Long, J., \& Li, C. (2017). Manufacturing quality prediction using intelligent learning approaches: A comparative study. Sustainability, 10(1), 85.

Blinco, L. J., Simpson, A. R., Lambert, M. F., Auricht, C. A., Hurr, N. E., Tiggemann, S. M., \& Marchi, A. (2014). Genetic algorithm optimization of operational costs and greenhouse gas emissions for water distribution systems. Procedia Engineering, 89, 509-516.

Bianchi, L., \& Dorigo, M. (2006). Ant colony optimization and local search for the probabilistic traveling salesman problem: a case study in stochastic combinatorial optimization. 
Chengbing, H., \& Xinxin, F. (2012). Institutions function and failure statistic and analysis of wind turbine. Physics Procedia, 24, 25-30.

Gao, Z., Cecati, C., \& Ding, S. X. (2015). A survey of fault diagnosis and fault-tolerant techniques-Part I: Fault diagnosis with model-based and signal-based approaches. IEEE Transactions on Industrial Electronics, 62(6), 3757-3767.

Hoel, M., \& Kverndokk, S. (1996). Depletion of fossil fuels and the impacts of global warming. Resource and Energy Economics, 18(2), 115-136.

Kahrobaee, S., \& Asgarpoor, S. (2010, September). Short and long-term reliability assessment of wind farms. In North American Power Symposium (NAPS), 2010 (pp. 1-6). IEEE.

Kusiak, A., \& Li, W. (2011). The prediction and diagnosis of wind turbine faults. Renewable Energy, $36(1), 16-23$.

Mensah, A. F., \& Dueñas-Osorio, L. (2012). A closed-form technique for the reliability and risk assessment of wind turbine systems. Energies, 5(6), 1734-1750.

Mingjun, L. I. U., Wenyuan, L. I., Juan, Y. U., Zhouyang, R. E. N., \& Ruilin, X. U. (2016). Reliability evaluation of tidal and wind power generation system with battery energy storage. Journal of Modern Power Systems and Clean Energy, 4(4), 636-647.

Nivedh, B. S. (2014). Major failures in the Wind Turbine components and the Importance of Periodic Inspection," http://www.windinsider.com/, 32-36.

Periola, A. A., \& Falowo, O. E. (2017). Cognitive communications for commercial networked earth observing fractionated small satellites. Wireless Personal Communications, 97(1), 443-467.

$\mathrm{Su}, \mathrm{C}$. , \& Hu, Z. (2018). Reliability assessment for Chinese domestic wind turbines based on data mining techniques. Wind Energy, 21(3), 198-209.

Simani, S., \& Farsoni, S. (2018). Fault Diagnosis and Sustainable Control of Wind Turbines: Robust Data-Driven and Model-Based Strategies. Butterworth-Heinemann.

Tabatabaeipour, S. M., Odgaard, P. F., Bak, T., \& Stoustrup, J. (2012). Fault detection of wind turbines with uncertain parameters: a set-membership approach. Energies, 5(7), 2424-2448.

Vogus, T. J., \& Welbourne, T. M. (2003). Structuring for high reliability: HR practices and mindful processes in reliability-seeking organizations. Journal of Organizational Behavior: The International Journal of Industrial, Occupational and Organizational Psychology and Behavior, 24(7), 877-903.

Wang, G. S., Huang, F. K., \& Lin, H. H. (2004, August). Application of genetic algorithm to structural dynamic parameter identification. In 13th World Conference on Earthquake Engineering, Vancouver (pp. 1-6).

Witczak, M., Rotondo, D., Puig, V., Nejjari, F., \& Pazera, M. (2018). Fault estimation of wind turbines using combined adaptive and parameter estimation schemes. International Journal of Adaptive Control and Signal Processing, 32(4), 549-567.

Witczak, M., Puig, V., Rotondo, D., de Rozprza Faygel, M., \& Mrugalski, M. (2015, June). A robust $\mathcal{H} \infty$ observer design for unknown input nonlinear systems: Application to fault diagnosis of a wind turbine. In Control and Automation (MED), 2015 23th Mediterranean Conference on (pp. 162-167). IEEE.

Zheng, M., \& Ming, X. (2017). Construction of cyber-physical system-integrated smart manufacturing workshops: A case study in automobile industry. Advances in Mechanical Engineering, 9(10), 1687814017733246.

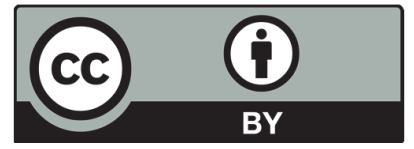

(C) 2019 by the authors; licensee Growing Science, Canada. This is an open access article distributed under the terms and conditions of the Creative Commons Attribution (CC-BY) license (http://creativecommons.org/licenses/by/4.0/). 